\title{
Applications of Tags in Multimodal Analysis of Motion Ergonomics for Healthcare Environments
}

\author{
Konrad Kluwak ${ }^{*}$, Marek Kulbacki ${ }^{* * * * * *}$, Anna Kolcz ${ }^{* * *}$ \\ *Wroclaw University of Science and Technology, Faculty of Electronics, Poland, \\ konrad.kluwak@pwr.edu.pl \\ **Polish-Japanese Academy of Information Technology, R\&D Center, \\ Koszykowa 86, 02-008 Warsaw, Poland, mk@pja.edu.pl \\ ***Wroclaw Medical University, Faculty of Health Science, Department of \\ Physiotherapy, Ergonomics and Biomedical Monitoring Laboratory, Grunwaldzka \\ 2, 50-355 Wroclaw,Poland, anna.kolcz-trzesicka@umed.wroc.pl \\ ****DIVE IN AI, Kamienna 15/54, 53-307 Wroclaw, Poland, info@diveinai.com
}

\begin{abstract}
This work introduces the Extracted Tags - EXTags, a short form of data extracted from a massive amount of multimodal human motion data for efficient human motion analysis. EXTags describe the only crucial space-time features from motion data in a certain period. We demonstrate how such brief representation might be a handful in an analysis of the patient transport situation from the point of view of the ergonomics of transporting people.
\end{abstract}

Keywords: Tag Detection; Motion Analysis; Ergonomics in Healthcare

\section{Introduction}

The transport of patients is a routine, frequently repeated activity performed by health professionals. Statistics show that routinely introduces terrible habits, which often accelerate employee occupational diseases (OHS) associated with the nonergonomic patient transfer. In 2017 American National Institute for Occupational Safety and Health reported over 1.25 trillion dollars loss due to the OHS problems [33]. In the daily activities of professional medical staff, few tasks do not require physical assistance. Many nurses must perform physically demanding patient care tasks that expose them to an increased risk of musculoskeletal disorders (MSD) and low-back disorders (LBD). A good survey [5] covering 132 papers on the frequency of injuries and musculoskeletal disorders pain concludes that nursing aides suffer from OHS.In [24], authors report a survey of 2405 nurses with range lifting tasks and confirm that low back pain is highly 
prevalent among nurses and is associated with a high-level sickness absence. Alamgir et al. in [1] examined injury claims that resulted in compensation or medical costs studying 2784 injury incidents and indicating the majority of injuries in nursing homes and acute care. Terrence et al. [28] examined the relationship between the regularity of patient lifting and the occurrence of back injuries and proved that regularity is a significant causative factor in the production of low back injuries in nursing personnel. Warming et al. [32] evaluated the inter-method reliability of a registration sheet for patient handling tasks and demonstrated that the logbook was reliable for both transfer and care tasks. In his systematic review [23] of papers between 2004 and 2016, Richardson concluded that additional research is necessary to identify interventions that may reduce the high rates of injury among nurses. Skotte in [25] investigated a lowback loading during everyday patient-handling tasks, emphasizing the compression and shear forces on L4/L5 joint and measured muscle activity. Hignett in [8] proved that nursing staff had lower levels of associated postural risk in positive safety cultures. Schnibye et al. [24] observed changes in mechanical load on the low-back and assessed a significant reduction was in spinal loading.

Literature studies show that for determining correct assessment methods, correct and incorrect lifting experiments should be carried out and registered in a controlled environment. Human Motion Laboratories for functional analysis equipped to measure kinematic, kinetic parameters, and electromyography of selected muscle groups involved in lifting are suitable for such trials. Efficient processing of particular information from heterogeneous massive multimodal human motion data (structured and unstructured) requires the availability of methods to select, process, and distinguish correct and incorrect samples of motion from the data stream in a fast way. Two popular mechanisms include indexing and tagging. However, an effective tagging of multiperson and multimodal human motion data requires an abstract description. The chosen motion modality or a few selected modalities or dependencies between them could be easily compared with other data samples in motion in the same time slot and modality.

There are several ideas in the literature on how to tag multimodal data. Rasiwasia et al. [22] demonstrated the benefits of joint text and image modeling by comparing the performance of a state-of-the-art image retrieval system to his image retrieval system, using the proposed joint model. A similar method was proposed by [27]. According to [9], current image segmentation approaches are divided into four categories: merging-based, splitting-based, statistical modelbased, and shot boundary classification-based. Authors claim that practical video structure analysis aims at segmenting a video and using semantic content. Atrey et al. [2] present known fusion strategies for combining multiple modalities appropriate to various tasks. In [3], the authors prove that the most appropriate is to use a taxonomy of multimodal machine learning with characteristic representation, translation, data fusion alignment, and co-learning in the 
multimodal world. Our proposition of EXTag representation is similar to [2] and [3] directions. We propose using several selected modalities simultaneously to represent particular motion patterns by single EXTag - Motion Tags (MT) entities. The ordered vector of Motion Tags represents multiperson motion as an instance of EXTag. We use hierarchical clustering, classification, and value derivative dynamic time warping [16] to compare normalized time series data efficiently.

To our best knowledge, current approaches consider only the analysis of artificial models or real situations without an accurate measurement methodology. In our case, the measurement concerns two people lifting supervised by professional physicians. In addition, researchers use dummy and artificial patients and simulations instead of accurate measurements of real people during activities.

Most of the existing approaches are based on a single domain, considering only the analysis of one movement feature to measure the correctness of lifting. Our main innovation introduces compound models that aggregate simultaneous activities of simple features like kinematic, kinetic, and EMG of selected body parts during motion. This experiment uses a person's motion which contains mutual relations lifter and being lifted person. The novelty starts from measurement protocol defined, which considers a lifting specialist and patient. We capture the details of the whole movement, taking into account the patient and the doctor, taking into account the kinetics, and on this basis, we build features, and these features are hierarchical. The second novelty starts from the measurement protocol defined, which considers lifting (nurse) and that the patient had been lifted.

\section{Measurement Methodology}

For demonstration purposes, the safe handling of the patient during the raising of a patient from lying to sitting in bed is being used. It requires two people, so it gives us complex data. The techniques of correct handling have been derived from the literature and verified by the experience of the employees of Wroclaw Medical University in Poland. Our study uses recorded multimodal information from 4 cameras, surface electromyography (sEMG), ground reaction forces (GRF), and full-body motion capture (MC). In multimodal observation, we focus on the configuration of hips, back, and knees and accompanying selected surface muscle tensions. 


\section{Activity Scenarios}

The activity scenario is composed of three phases: 1) preparing the patient for movement, 2) turning the patient to lie down on his side, in a safe position, 3) moving the patient from a safe position to a seated position at the edge of the bed.

Correct lifting must follow the rules of the loads lifting mechanics:

- $\quad$ keep the spine in a neutral position (in a slight squat),

- bend of knees and hips (knees-patellas should not exceed the line of fingers),

- hold the patient close to the body at waist height (arms not extended),

- move as smoothly as possible.

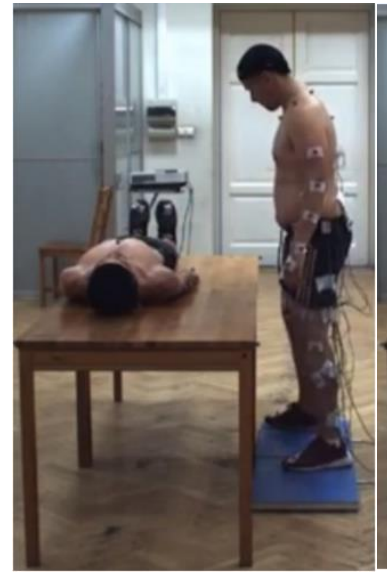

(a) 1.1

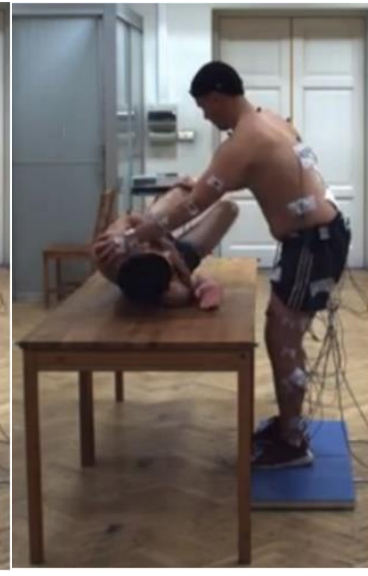

(b) 2.3

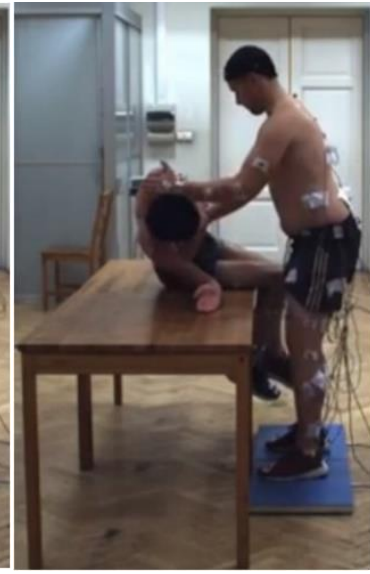

(c) 3.2

Figure 1

Key phases of patient lifting (numbering as described in activity scenario procedure)

The recommended patient transfer techniques, including a patient who is heavier than the caregiver, without overloading the spine and exposing him/her to injuries, are described in detail:

\section{Prepare the patient for movement}

1.1. Position yourself facing the bed on which the patient is lying. Take a relaxed position.

1.2. Bend your knees slightly, position your feet freely, tighten your torso is stabilizing muscles, Put your spine in a safe position, breathe freely. 
1.3. Get closer to the patient's head, now change the position of the feet. Both feet are pointing towards the patient's head. Forward, leg closer to the patient's head, forward-facing, bent in the knee, bodyweight transferred to the front leg, rear leg slightly bent, leg and trunk muscles tense, spine in a safe position, free breathing.

1.4. Move the patient's hand closer to the edge of the bed, bend it at the elbow, and then bend it at the elbow. Slide your hand under your head (pillow). Point the patient's head towards the hand.

1.5. Move the patient's other hand over the chest to the edge of the bed and put your hand on the bed.

1.6. Move closer to the patient's feet, now change the position of the feet, both feet forward-facing, leg closer to the patient's legs forwardfacing, curved in the knee, bodyweight transferred to the front leg, rear leg slightly bent, leg muscles and your torso is tense, your spine is in a safe position, breath freely.

1.7. Slide your hand closer to the patient's lying foot and bend your leg by slightly sliding it over the mattress. Then, bend the patient's other leg in the knee.

\section{Turn the patient to lie down on his side in a safe position.}

2.1. Move closer to the patient's torso.

2.2. Stand in front of the patient, slightly bend your knees, position your feet freely, one leg remains in front, bent in the knee, the other remains in the back, the bodyweight remains on the front leg, tighten the muscles stabilizing the torso, put the spine in a safe position, breathe freely.

2.3. Place one hand on the patient's shoulder, do not bend the hand in the wrist, place the other hand near the patient's hip, slowly move the body weight from the front leg to the rear leg, turning the patient to lie on his side, in a safe position.

3. Move the patient from a safe position to a seated position at the edge of the bed.

3.1. Change the position of your feet. Position yourself facing the bed on which the patient is lying, take a relaxed position, slightly bend your knees, put your feet forward freely, fingers slightly to the sides, tighten the torso stabilizing muscles, put your spine in a safe position, breathe freely. Move the patient's legs towards the edge of the bed, slowly lowering them out of bed. Secure the patient's legs with your leg. 
3.2. Ask the patient to start pushing away from the bed with hand, and at the same time lightly support the patient's movement with hands so that the patient changes position from lying on the side to sitting on the bed with legs down.Support the patient's hands on the bed, on both sides of the patient's body. If possible, the patient's feet should be supported (e.g., on the floor).
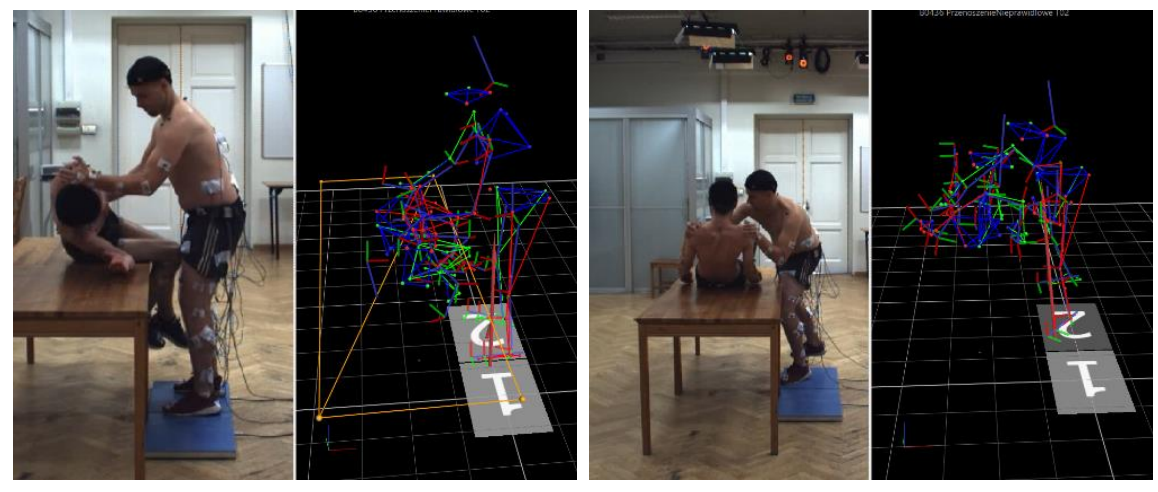

Figure 2

Multimodal Visualization of correct (left side) and incorrect (right side) patient lift with video, 3D motion, and position on the force plates

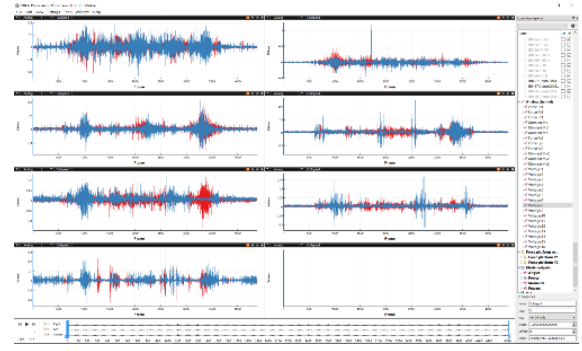

(a) Correct

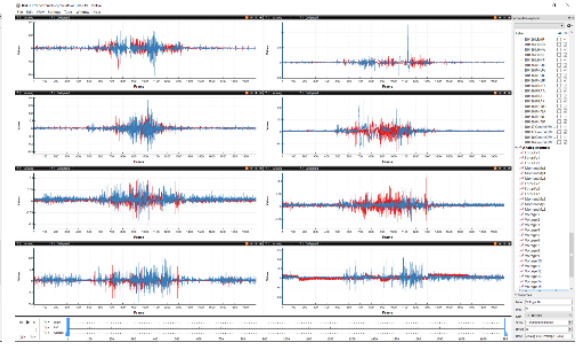

(b) Incorrect

Figure 3

Examples of correct and incorrect registrations of selected muscle tensions during lifting procedure

In addition, for each actor, ten squats were registered with and without a load to perform them correctly and incorrectly. The correct motion applies to the rules written above. The incorrect does not apply. This exercise was performed as a reference to EMG measurements of patient lifting. 


\section{Measurement Configuration}

Reference database has been registered using the system for multimodal motion acquisition in Human Motion Laboratory (HML) of the Polish-Japanese Academy of Information Technology (PJAIT) in Bytom, Poland. Laboratory provides a comprehensive environment for multimodal data acquisition, management, and analysis. It allows motion data acquisition by synchronous, simultaneous measurement and recording of video streams, muscle potentials, motion kinematics, and kinetics. It makes it possible to apply a spatio-temporal correlation between the acquired data. MX-Giganet Lab is responsible for hardware synchronization during data acquisition from base systems. Our particular multimodal recordings contain:

- 3D body motions of 2 actors (patient and nurse) were registered based on Vicon's Motion Kinematics Acquisition and Analysis System equipped with 28 Motion Capture Camera (10 MX T40, 10 Bonita 10, and 8 Vantage 5). The system registers 39 reflective markers on each of the actors (tracked at $100 \mathrm{fps}$ ) based on sets for the Plug-in Gait full-body model and provides information on the location of all skeleton joints. Markers were placed on significant body segments: 4 on Head, five on Torso, 14 on the left and right side of upper limbs, and 16 on left and right side of the lower body according to Vicon specification [31].

- Muscle potentials for nurses by Noraxon's Dynamic Electromyography (EMG) System allows for 16-channel measurement of non-gel electrodes in compliance with the SENIAM guidelines. For registration EMG, has been defined measurement configuration (Fig. 4). EMG electrodes measure the work of muscles taking part in stabilizing the figure of the person who is lifting the patient.

- Ground Reaction Forces (GRF) were recorded (1000 Hz) for the nurse's body by Kistler Force Plates (Internal Amplifier), two dynamometric platforms with equal accuracy on the entire surface of the platform at $1000 \mathrm{~Hz}$ frequency. The system has been used to check on which leg is the more significant body pressure.

- Video system: using multi-camera video system for simultaneous recording from 3 Full HD $(25 \mathrm{~Hz})$ cameras and lossless video recording equipped with Basler's pilot piA1900-32gm/gc GigE Vision. The cameras recorded the view from the front, back, and side positions of the scene. Recordings contain information about the position of actors over time. 


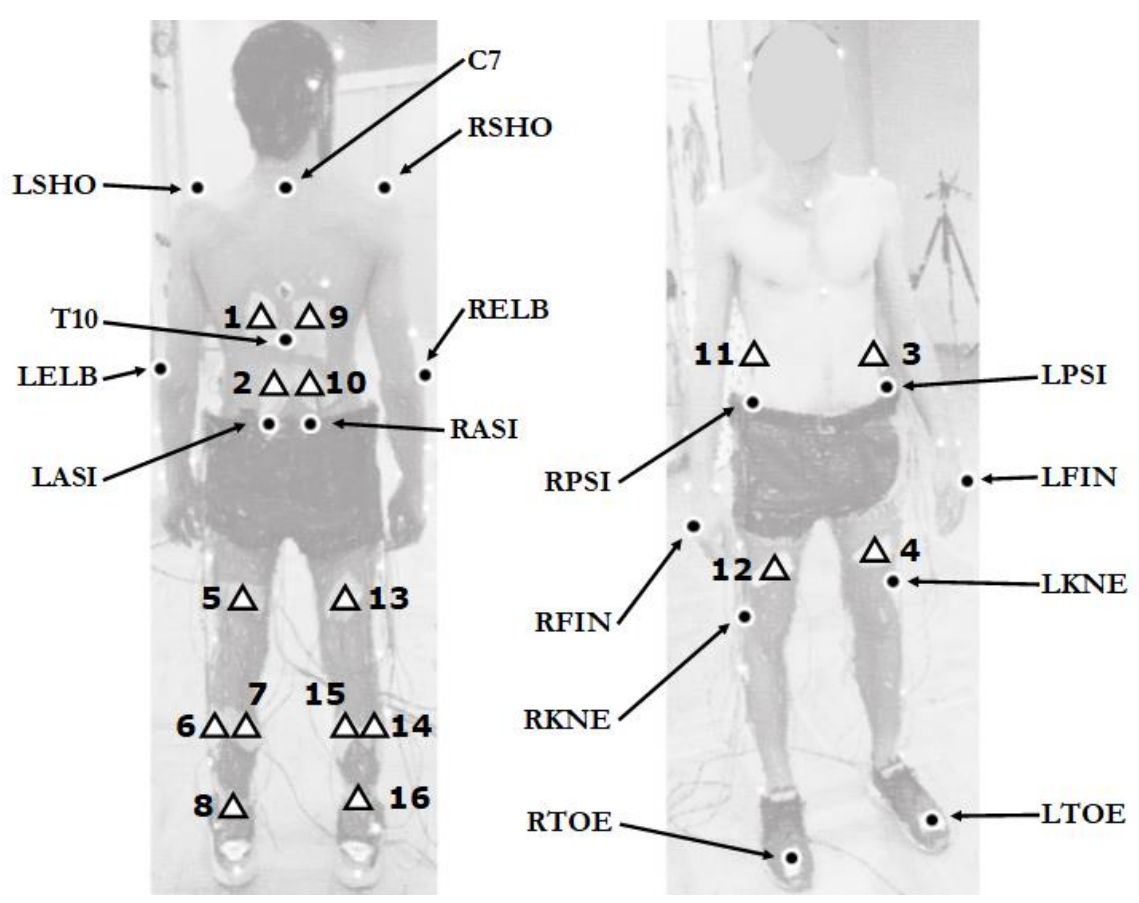

Figure 4

Lifting actor with EMG electrodes and skeleton markers configurations

Hardware synchronization and hardware calibration were described in the paper [17]. Each multimodal recording has a single C3D file containing kinematic and kinetic motion parameters, three video streams, and configuration files. In addition to patient transfer, other movements were recorded to determine maximum muscle tension in a similar situation. Two actors have been recorded performing the movements representing correct and incorrect lifting (Fig. 2). This multimodal measurement configuration has 414 distinctive motion parameters in data records, resulting in a large amount of data.

The dataset with patient lifting (DPLP) has been made public for scientific research purposes according to the initiative of Living Labs for Human Motion Analysis and Synthesis in Shared Economy Model. It is available in the resources of the R\&D Center PJAIT: https://res.pja.edu.pl (accessed on 1 July 2021). 


\section{Extracted Tag Concept}

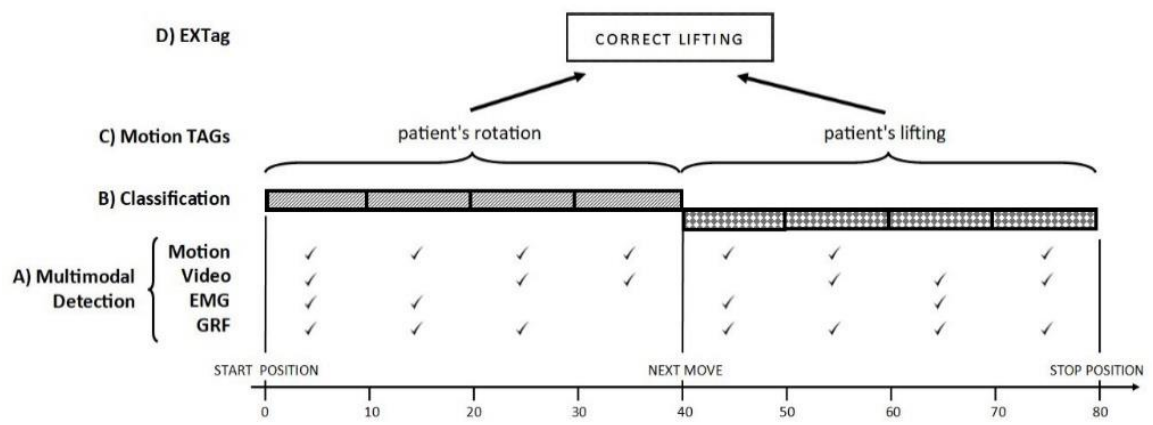

Figure 5

EXTag concept with four level of operations

To reduce data amount and faster operations, we introduce the Extracted Tag (EXTag) concept. It will allow on the performance of multimodal assessment and analysis by: (A) event detection in multimodal data, (B) ranking motion recognition, (C) Motion Tag classification, and (D) EXTag marking. These operations allow quickly performing content-based motion retrieval from complex multidimensional and multimodal space. EXTag is a part of a hierarchical representation of multimodal motion information composed of an ordered sequence of motions - Motion Tags. An example of the EXTag representation in graphical form demonstrating the patient lifting task is shown in Figure 5. EXTag is assigned to the appropriate class after the schema elements of the defined Motion Tag sequence are correctly recognized. Motion Tag is a concise, minimal representation of a given segment of motion measurement, which consists of characteristic data blocks of the selected motion. Motion Tag reference is considered as a part of multimodal measurements. In multimodal measurements, we set the characteristics of given modalities by labels: correct, incorrect. These characteristics are not the whole body configuration but the only configuration of the distinguished fragments. In multimodal motion feature space, selected features that are valid for specific Motion Tags are considered. They are applied to the Motion Tag subspace and determine whether a given Motion Tag is complete.

In addition, an order in which the given movements occur is maintained. The correct occurrence of Motion Tag determines the resulting EXTag. In this way, building a minimal representation consisting of labels and selected sets of values for specific, selected quantities during movement. These quantities are described in a more abstract way compared to the raw data recorded in the laboratory. An example of such an abstraction in our case of patient lift is the position of the arms relative to the hips, described as the mutual position of several joints relative to each other. It is not an anatomical norm but an accepted scheme that unambiguously defines the correct lifting movement in the patient lifting scheme. EXTag is the minimum representation that eliminates the need to 
search the entire movement space using a data fragment as a movement pattern. It is a high-level representation that does not penetrate into the detailed structure of each of the measured objects. In the case of two people's movements, the specific properties of these movements are measured in a given time. Patterns of these movements are also considered elements of measurements. The minimal representation described using EXTag consists of Motion Tags representing single motions. Every single movement is represented by specific modality values measured in time.

Tag Modelling Algorithm:

1) Define the pattern of movement - Motion Tag using motion features selected by a domain expert. Such a pattern describes the average duration of a defined movement, the properties of this movement described by changes in the kinematics and scopes of angles of joints (degrees of freedom) in the skeletal model, and at the same time changes in kinetics and muscles activities in the muscle model.

2) Select the dominant feature of the defined Motion Tag.

3) Unify a Tag for a specific modality (kinematics, EMG).

4) Collect all the characteristics of a given Motion Tag.

\section{Extracted Tag Application}

Motion Tag is correctly recognized only when the previously defined motion blocks represented by different modalities occur in a specific order. For that, we propose a logistic regression classifier with compressed sparse row representation that gives optimal performance. The block size depends on the specific motion description and required features selected from the corresponding measurements. Depending on the motion recording system used, the motion block may include motion capture, EMG, GRF, video, and other required modalities. The correct recognition takes place for the optimal feature vector of ordered motion states appear on multimodal data. In our multimodal data for the definition of minimal distinguishable motion representation at first, two data modalities: EMG and motion capture have been considered, which requires 70 parameters in total and results in 6 times fewer data and feature space.

In our example, we measure the motion of two people, but in general, we have $P$ people in multiperson motion. EMG features for a person $\mathrm{P}_{\mathrm{n}}, \mathrm{n} \in\{1 . . \mathrm{N}\} \mathrm{P}_{\mathrm{n}}, \mathrm{n} \in$ $\{1 . . \mathrm{N}\}$ are given by Integral Absolute Value calculated for a particular $E M G_{i}$ channel represented by $x_{j}$ samples in window $K$ :

$$
E M G_{P_{n}}=\left\{E M G_{1}, \ldots, E M G_{16}\right\}, \quad E M G_{i}=\frac{1}{K} \sum_{j=(i-1) * N+1}^{i * K}\left|s_{j}\right|
$$


In the discussed example, for the correct lifting movement, we expect EMG modality of all muscles at a constant level and low values compared to the intense muscle load when lifting a significant weight. In the abnormal lifting movement, EMG reaches high values when the nurse overturns and lifts the patient. This stroke is identified as a threat to the spine. In order to define the optimal EMG vector, we additionally check if the tension on the back is less than on the abdomen and legs. High tension on the abdomen is identified as correct, high tension on the muscles of the squares is identified as incorrect. We do not know specific EMG values, but we rely on signal variation or a standardized pattern for a specific person based on correct and incorrect examples.

Kinematic features for person $\mathrm{n}-\mathrm{KF}_{\mathrm{P}_{\mathrm{n}}} \mathrm{n}-\mathrm{KF}_{\mathrm{P}_{\mathrm{n}}}$, are defined by set of $\mathrm{cc}$ geometric relations between specified skeleton configurations creating spatial relations in the window KK:

$$
K F_{P_{n}}=\left\{K F_{1}, \ldots, K F_{c}\right\}, \quad K F_{\{i\}}=\left\{\sum_{j=1}^{k k}\left(w_{j} * v_{j}\right)\right\}
$$

where kkkk is the feature dimension of particular geometric relation, $\mathrm{v}_{\mathrm{j}} \mathrm{v}_{\mathrm{j}}$ particular feature and $w_{j} w_{j}$ normalized weight for the $j \mathrm{j}$-th geometric feature.

Here we refer directly to the description of the movement from the previous chapter. In our observation, we are focused on the particular configuration of hips, back and knees. Due to the limited data, we study the rotation of the spine during lifting (hip line relative to the shoulder line), lifting work with hands close to the body. The bending of the nurse's legs in the knees when lifting reduces the lever, reducing required parameters to 32 dimension feature vector. For window KK for normalization and comparison of coherent kinematic and EMG features we use VDDTW [16] following agglomerative clustering for partitioning of mixed multimodal motion feature set into distinguished groups of primitive multimodal motions according to the distribution of generic model $\mathrm{GM}_{\mathrm{i}}, \mathrm{i} \in\{\mathrm{T}, \mathrm{F}\} \mathrm{GM}_{\mathrm{i}}, \mathrm{i} \in$ $\{\mathrm{T}, \mathrm{F}\}$ :

$$
g_{i, l}\left(s_{l}(m)\right)=\frac{1}{\sqrt{v_{l} 2 \pi}} e^{\frac{-\left[s_{l(m)}-a v_{l}\right]^{2}}{2 v_{l}}}
$$

For classification purposes we use similarity measure and classify multimodal motion features according to similarity measure with maximal value:

$$
h(s(m))=\underset{i \in\{1,2, \ldots, K\}}{\operatorname{argmax}}\left[P\left(G_{i}\right) \prod_{l=1}^{M} w_{l} g_{i, l}\left(s_{l}(m)\right)\right]
$$

For example, we assumed a limited set of different motions, and each group $G_{i} G_{i}$ has the same likelihood $P\left(G_{i}\right)=\frac{1}{K} P\left(G_{i}\right)=\frac{1}{K}$. 


\section{Conclusions}

In this paper, we introduced an important problem of lifting procedure as an example of massive multiperson, multimodal motion data and elements defining the EXTags allowing for the unequivocal determination of the correctness of movement performed by medical personnel during the lifting of the patient. There was proposed reduced motion description by EXTags introduction, understood as a configuration of feature subset from a set of multimodal quantities relating to the description of motion on the high level extracted from the recorded human motion. The experiment demonstrated a significant difference between the normal and abnormal lifting characterized by limited and selected features among different motion parameters in different time slots. Such motion patterns defined for lifting a patient performed in a certain period were described by EXTag and given by specifications of multimodal motion configuration. In our study, the activity scenario and experiment description were proposed. Additionally, a multimodal measurement configuration and its impact on data quantity were presented, including the EXTag concept and its actual usability. Due to the limit of pages, the article has been significantly reduced and another paper focused on the experiment results is planned. The work will be further developed towards the analysis of human motion data for medical applications. The EXTag concept will be tested for other domains with multimodal motion data. In the future, we are considering conducting extensive experiments with different methods and models, which proved to be successful in modeling systems in various fields as presented in [4] [7] [12] [20] [34].

\section{Acknowledgements}

This study was supported by the Scientific Research from Polish-Japanese Academy of Information Technology, R\&D Center and DIVE IN AI and statutory funds of Wroclaw University of Science and Technology, Faculty of Electronics and Wroclaw Medical University, Department of Physiotherapy.

\section{References}

[1] H. Alamgir, Y. Cvitkovich, S. Yu, and A. Yassi, "Work-related injury among direct care occupations in British Columbia, Canada," Occup. Environ. Med.

[2] P. K. Atrey, M. A. Hossain, A. El Saddik, and M. S. Kankanhalli, "Multimodal fusion for multimedia analysis: A survey," Multimed. Syst., Vol. 16

[3] T. Baltrusaitis, C. Ahuja, and L. P. Morency, "Multimodal Machine Learning: A Survey and Taxonomy," IEEE Trans. Pattern Anal. Mach. Intell., Vol.

[4] W. Y. Cheng and C. F. Juang, "A fuzzy model with online incremental SVM and margin-selective gradient descent learning for classification problems,” IEEE Trans. Fuzzy Syst., Vol. 22, No. 2, pp. 324-337, 2014 
[5] K. G. Davis and S. E. Kotowski, "Prevalence of Musculoskeletal Disorders for Nurses in Hospitals, Long-Term Care Facilities, and Home Health Care: A Comprehensive Review," Human Factors, Vol. 57, No. 5. SAGE Publications Inc., pp. 754-792, 28-Aug-2015

[6] M. Fray and S. Hignett, "Using patient handling equipment to manage mobility in and around a bed.," Loughborough University, Jan. 2015

[7] E. L. Hedrea, R. E. Precup, and C. A. Bojan-Dragos, "Results on tensor product-based model transformation of magnetic levitation systems," Acta Polytech. Hungarica, Vol. 16, No. 9, pp. 93-111, 2019

[8] S. Hignett and E. Crumpton, "Competency-based training for patient handling," Appl. Ergon., Vol. 38, No. 1, pp. 7-17, Jan. 2007

[9] W. Hu, N. Xie, L. Li, X. Zeng, and S. Maybank, "A survey on visual content-based video indexing and retrieval," IEEE Trans. Syst. Man Cybern. Part C Appl. Rev., Vol. 41, No. 6, pp. 797-819, 2011

[10] M. Jäger et al., "Lumbar-load analysis of manual patient-handling activities for biomechanical overload prevention among healthcare workers," Ann. Occup. Hyg., Vol. 57, No. 4, pp. 528-544, 2013

[11] C. Jordan, A. Luttmann, A. Theilmeier, S. Kuhn, N. Wortmann, and M. Jäger, "Characteristic values of the lumbar load of manual patient handling for the

[12] C. F. Juang, Y. Y. Lin, and R. B. Huang, "Dynamic system modeling using a recurrent interval-valued fuzzy neural network and its hardware implementation," Fuzzy Sets Syst., Vol. 179, No. 1, pp. 83-99, 2011

[13] K. Kindblom, Movement awareness and communication in patient transfer : An educational intervention. 2009

[14] K. Kjellberg, Work technique in lifting and patient transfer tasks. 2003

[15] P. Konrad, The ABC of EMG: a practical introduction to kinesiological electromyography. Noraxon USA, Inc, 2006

[16] M. Kulbacki and A. Bak, "Unsupervised Learning Motion Models Using Dynamic Time Warping," in Intelligent Information Systems 2002, Springer, 2002

[17] M. Kulbacki, J. Segen, and J. P. Nowacki, “4GAIT: Synchronized MoCap, video, GRF and EMG datasets: Acquisition, management and applications," 2014

[18] R. J. Parkinson, M. Bezaire, and J. P. Callaghan, "A comparison of low back kinetic estimates obtained through posture matching, rigid link modeling and

[19] M. Piorek, Analysis of Chaotic Behavior in Non-linear Dynamical Systems, Vol. 160, 2019 
[20] C. Pozna and R. E. Precup, "Applications of signatures to expert systems modelling," Acta Polytech. Hungarica, Vol. 11, No. 2, pp. 21-39, 2014

[21] R. E. Precup, T. A. Teban, A. Albu, A. B. Borlea, I. A. Zamfirache, and E. M. Petriu, "Evolving Fuzzy Models for Prosthetic Hand Myoelectric-Based Control,” IEEE Trans. Instrum. Meas., Vol. 69, No. 7, pp. 4625-4636, 2020

[22] N. Rasiwasia et al., "A new approach to cross-modal multimedia retrieval," in MM'10 - Proceedings of the ACM Multimedia 2010 International Con

[23] A. Richardson, B. McNoe, S. Derrett, and H. Harcombe, "Interventions to prevent and reduce the impact of musculoskeletal injuries among nurses: A system

[24] B. Schibye, A. F. Hansen, C. T. Hye-Knudsen, M. Essendrop, M. Böcher, and J. Skotte, "Biomechanical analysis of the effect of changing patienthandling

[25] J. H. Skotte, M. Essendrop, A. F. Hansen, and B. Schibye, "A dynamic 3D biomechanical evaluation of the load on the low back during different patient-ha

[26] J. Smedley, P. Egger, C. Cooper, and D. Coggon, "Manual handling activities and risk of low back pain in nurses," Occup. Environ. Med., Vol. 52

[27] C. G. M. Snoek and M. Worring, "Multimodal video indexing: A review of the state-of-the-art," Multimedia Tools and Applications, Vol. 25, No. 1

[28] T. J. Stobbe, R. W. Plummer, R. C. Jensen, and M. D. Attfield, "Incidence of low back injuries among nursing personnel as a function of patient lifting

[29] M. Strohmaier, C. Körner, and R. Kern, "Understanding why users tag: A survey of tagging motivation literature and results from an empirical study,"

[30] N. Ulutasdemir and F. Tanir, "Occupational Risks of Health Professionals," in Occupational Health, InTech, 2017

[31] Vicon Motion Systems Limited, "Full Body Modeling with Plug-in Gait," Plug-in Gait Reference Guide, 2017

[32] S. Warming, D. H. Precht, P. Suadicani, and N. E. Ebbehøj, "Musculoskeletal complaints among nurses related to patient handling tasks and psychosocial $\mathrm{f}$

[33] N. Wiggermann, "Biomechanical Evaluation of a Bed Feature to Assist in Turning and Laterally Repositioning Patients," Hum. Factors, Vol. 58, No.

[34] R. Zall and M. R. Kangavari, "On the construction of multi-relational classifier based on canonical correlation analysis,” Int. J. Artif. Intell., Vol. 17, No. 2, pp. 23-43, 2019 